\title{
Resistencia a la Fractura de Dientes Tratados Endodónticamente Obturados con Selladores Biocerámicos Versus Selladores Resinosos. Revisión Sistemática
}

\author{
Fracture Resistance of Endodontically Treated Teeth Obturated with \\ Bioceramic Sealers and Epoxy Resin-Based Sealers. A Systematic Review \\ Luis Felipe Morales Cáceres'; Samuel Ignacio Reyes Montenegro'; \\ Silvio Jesús Álvarez Vanegas ${ }^{2}$ \& Scarlette Hernández-Vigueras ${ }^{2,3}$
}

MORALES, C. L. F.; REYES, M. S. I.; ÁlVAREZ, V. S. J. \& HERNÁNDEZ-VIGUERAS, S. Resistencia a la fractura de dientes tratados endodónticamente obturados con selladores biocerámicos versus selladores resinosos. Revisión sistemática. Int. J. Odontostomat., 13(1):31-39, 2019.

RESUMEN: El objetivo de este estudio fue determinar mediante la revisión de la literatura científica disponible cuál es el tipo de cemento sellador que proporciona mayor resistencia a la fractura en dientes tratados endodónticamente, los cementos a base de biocerámicos o los en base a resina epóxica. Se realizó una revisión sistemática de acuerdo a las bases de los estamentos de PRISMA, en las bases de datos Medline, SciELO, Trip Database, LILACS, Web of Science, Cochrane. Se realizó la búsqueda a 10 años y se incluyeron sólo estudios in vitro. Se encontraron 202 artículos, luego se eliminaron los artículos duplicados y se excluyeron los estudios no atingentes por título y resúmenes, quedando ocho artículos que se revisaron a texto completo. En esta etapa se excluyeron dos estudios. Un total seis estudios fueron incluidos en esta revisión. De estos, ninguno encontró diferencia significativas entre cementos selladores a base de biocerámicos y a base de resina epóxica, en cuanto a resistencia a la fractura de los dientes tratados endodónticamente. Sin embargo, en 4 estudios los valores más altos encontrados de resistencia a la fractura estuvieron dados por los selladores en base a resina epóxica. Los artículos seleccionados, teniendo en consideración las limitaciones propias de los estudios in vitro, concluyen que en cuanto a resistencia a la fractura no hay diferencias significativas entre el uso de cementos selladores a base de biocerámicos y cementos selladores a base de resina epóxica en la obturación radicular de dientes tratados endodónticamente.

PALABRAS CLAVE: cementos biocerámicos, resistencia a la fractura, diente tratado endodónticamente, obturación radicular.

\section{INTRODUCCIÓN}

Las fracturas radiculares son la tercera causa más común de pérdida de dientes después de caries dental y enfermedad periodontal. La prevalencia de dichas fracturas en dientes tratados endodónticamente (DTE) se encuentra entre el 2 y el $20 \%$, mientras que el $94 \%$ de los dientes con fracturas radiculares han tenido antecedentes de tratamiento endodóntico (Chang et al., 2016; García-Guerrero et al., 2018).
En endodoncia, para lograr un tratamiento exitoso a largo plazo, la obtención de un sellado hermético, junto con la limpieza y la configuración del conducto radicular son unas de las claves (Schilder, 1967). En cuanto a la configuración del conducto, si bien es necesaria la instrumentación del conducto radicular, varios estudios han indicado que la remoción de la estructura al interior de éste, disminuye la resistencia

\footnotetext{
${ }^{1}$ Escuela de Odontología, Universidad Austral de Chile, Chile.

2 Especialista en Endodoncia, Instituto de Odontoestomatología, Facultad de Medicina, Universidad Austral de Chile, Chile.

${ }^{3}$ Profesor Auxiliar, Facultad de Medicina, Universidad Austral de Chile, Chile.

Patrocinio: Este trabajo fue patrocinado por la Escuela de Odontología, Facultad de Medicina, Universidad Austral de Chile, Chile.
} 
MORALES, C. L. F.; REYES, M. S. I.; ÁLVAREZ, V. S. J. \& HERNÁNDEZ-VIGUERAS, S. Resistencia a la fractura de dientes tratados endodónticamente obturados con selladores biocerámicos versus selladores resinosos. Revisión sistemática. Int. J. Odontostomat., 13(1):31-39, 2019.

a la fractura del diente, sin importar si la instrumentación es manual o mecanizada (Lam et al., 2005).

La instrumentación del conducto radicular es un paso esencial en el tratamiento endodóntico. Se entiende que a medida que se retira la dentina durante la fase de instrumentación, el efecto de debilitamiento en la raíz es inevitable. Por lo tanto, es necesaria la incorporación de un material dentro del conducto para reducir este debilitamiento, el cual debe ser capaz de reforzar y fortalecer la estructura debilitada, además de sellar el canal radicular, logrando así un sellado hermético tridimensional (Ashraf et al., 2013). Si bien la gutapercha es el gold estándar dentro de los materiales de obturación radicular, es necesaria además la utilización de un material sellador para asegurar el sellado apical. Las propiedades de un sellador ideal incluyen la creación de un sello apical resistente a las bacterias, poseer actividades antimicrobianas, ser tolerante a los tejidos, y proporcionar buena adhesión entre sí y la dentina intrarradicular. Hasta hace pocos años, el material más utilizado eran los selladores en base a resina epóxica, pero los materiales biocerámicos han sido recientemente introducidos como materiales selladores en endodoncia. Los biocerámicos son materiales cerámicos diseñados específicamente para su uso en medicina y odontología. Estos materiales contienen principalmente: Alúmina, zirconia, vidrio bioactivo, cerámica de vidrio, revestimientos, hidroxiapatita y fosfatos de calcio reabsorbibles (Malhotra et al., 2014). Los selladores biocerámicos son biocompatibles, no se contraen y químicamente son estables dentro del entorno biológico. También tienen la capacidad de formar hidroxiapatita durante el proceso de fraguado $y$, en última instancia, crear un vínculo entre la dentina y el material de relleno.

En una revisión sistemática sobre las propiedades físico-químicas y biológicas de los cementos selladores a base de bioceramicos, se concluyó que la fuerza de adhesión, radiopacidad, $\mathrm{pH}$, solubilidad, tiempo de trabajo, cambios dimensionales, fluidez y liberación de iones de calcio de dichos cementos son iguales o mejor a los cementos a base de resina epóxica (Silva Almeida et al., 2017).

Por lo anteriormente señalado, surge la inquietud de que si los cementos selladores a base de biocerámicos, además de entregar todas estas propiedades al DTE, le otorgan a este último, mejor resistencia a la fractura que los cementos selladores a base de resina epóxica. El objetivo de este estudio es revi- sar la literatura actual comparando la resistencia a la fractura aportada al DTE por los cementos selladores en base a resina epóxica versus los nuevos cementos selladores en base a biocerámicos.

\section{MATERIAL Y MÉTODO}

Esta revisión sistemática se llevó a cabo de acuerdo a las bases de los estamentos de PRISMA (Moher et al., 2009). Se realizó una revisión sistemática de la literatura de artículos in vitro para responder la pregunta PICO, donde P fueron los DTE; la intervención fue la obturación radicular con cementos selladores; la comparación fue entre cementos selladores a base de biocerámicos versus los cementos selladores a base de resina epóxica; y por último el resultado esperado es la resistencia a la fractura aportadas al diente por los diferentes cementos selladores registrada en Newtons.

Búsqueda electrónica: fue realizada por los autores en los siguientes buscadores y bases de datos: Medline, SciELO, Trip Database, LILACS, Web of science y Cochrane. La búsqueda fue llevada a cabo con límite de 10 años hasta julio del 2017. Las estrategias de búsqueda utilizada en cada buscador esta descrita en la Tabla I. Además los autores hicieron una búsqueda manual de la literatura en busca de estudios relevantes no encontrados con dichas estrategias.

Criterios de inclusión: Estudios in vitro, donde las muestras fueran dientes sanos sometidos a tratamientos endodónticos para luego ser obturados con cementos selladores biocerámicos y cementos a base de resina epóxica. Estudios donde se mida la resistencia a la fractura a través de fuerzas compresivas aplicadas sobre los dientes obturados con los selladores a base de biocerámicos y a base de resina epóxica. Estudios que posean grupo control.

Criterios de exclusión: Estudios que no evalúen la resistencia a la fractura con máquinas universales de testeo. Estudios que incluyan fuerzas no compresivas para evaluar la resistencia a la fractura. Estudios que expresen sus resultados en medidas que no sean Newtons $(\mathrm{N})$ y estudios que declaren conflictos de interés.

Colección y análisis de datos: Los resultados de cada búsqueda fueron ingresados en una hoja de cálculo de Google Drive (@2017 Google) donde los auto- 
res de manera manual revisaron los artículos en busca de los estudios duplicados. Luego de esto, se realizó una selección de artículos de acuerdo a los criterios de inclusión y exclusión, en primer lugar se analizaron los títulos y resúmenes de los estudios, y luego los estudios pertinentes se revisaron a texto completo. Esto se llevó a cabo de manera independiente por cada autor y luego se compararon los resultados, los estudios en conflicto se analizaron por los autores y se llegó a un consenso para su inclusión dentro de la revisión.

Extracción y manejo de datos: Para la extracción y manejo de los datos los artículos seleccionados fueron leídos nuevamente y se realizó la extracción de los datos de acuerdo a los siguientes parámetros: Autor y año, tipo de muestra, selladores utilizados, grupo experimentales de los estudios, valores de resistencia a la fractura obtenidos, y por último resultados de cada estudio. Los datos fueron tabulados en una tabla creada a través de la plataforma Google Docs de Google Drive (@2017 Google).

Evaluación del riesgo de sesgo en los estudios incluidos: Para analizar la calidad de los estudios in- cluidos en la revisión se les evaluó el riesgo de sesgo a cada uno de acuerdo con los siguientes parámetros para estudios in vitro: aleatorización de los dientes, uso de dientes sin caries/cracks/anomalías, uso de materiales según las instrucciones del fabricante, uso de dientes de dimensiones similares, descripción del cálculo del tamaño de muestral, cegamiento del operador de la máquina de testeo y uso de medio de conservación. Si los autores informaron el parámetro, el estudio obtenía un signo positivo (+) en ese parámetro. En caso contrario, si no fue posible encontrar la información, el artículo obtenía un signo negativo (-). Los estudios que informaran de uno a tres parámetros se clasificaban como de alto riesgo de sesgo, cuatro o cinco parámetros como riesgo moderado de sesgo y seis o siete parámetros como de bajo riesgo de sesgo. Esta evaluación de los parámetros del riesgo de sesgo se adaptó de estudios previos con características similares (Sarkis-Onofre et al., 2014; Sedrez-Porto et al., 2016).

Los datos faltantes de uno de los estudios (Upadhyay et al., 2017) fueron enviados por el autor del estudio previa solicitud vía mail por parte de los autores de la revisión.

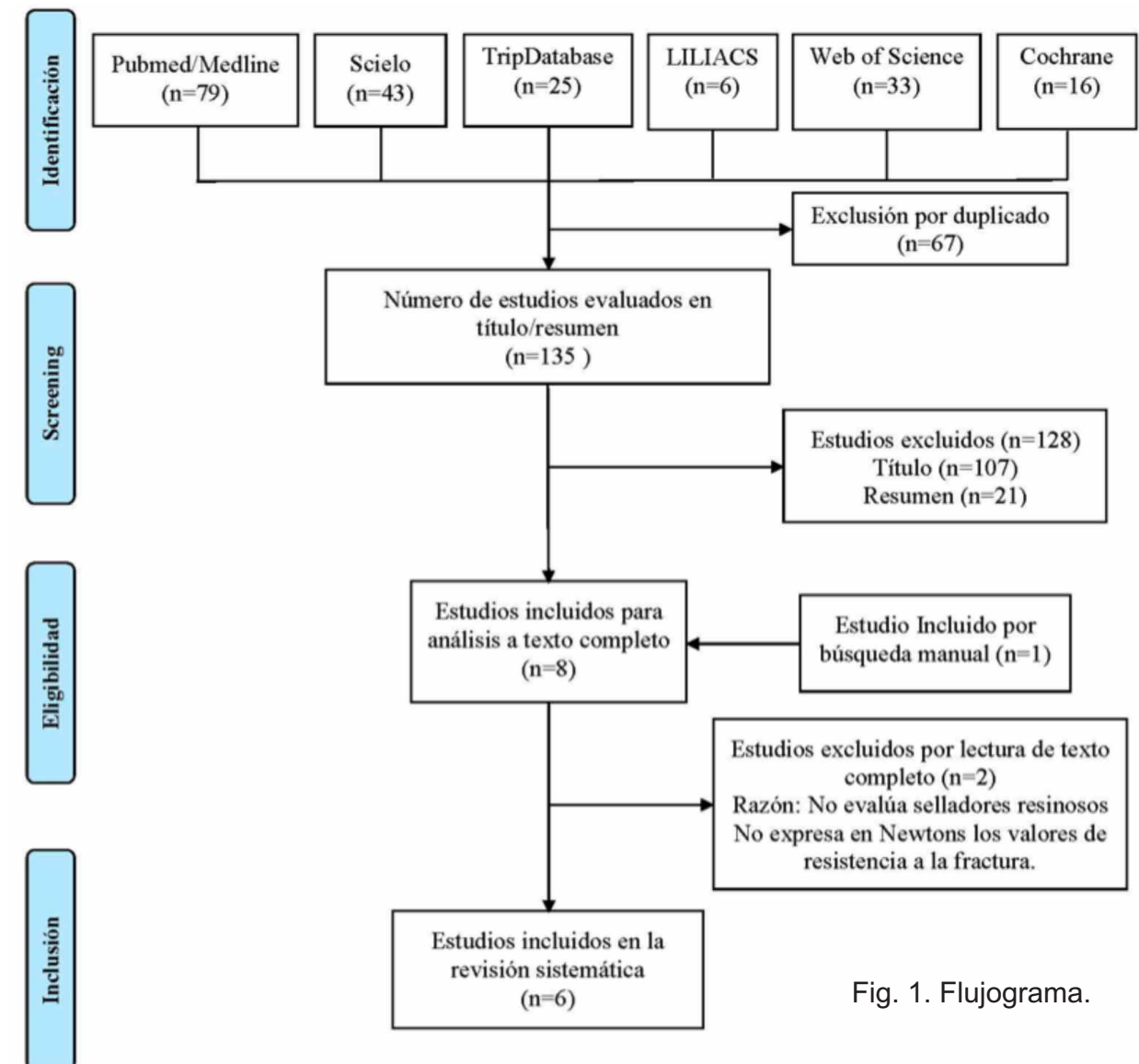


Tabla I. Estrategias de búsqueda.

\begin{tabular}{llc}
\hline Base de datos & \multicolumn{1}{c}{ Estrategia de búsqueda } & Resultados \\
\hline Medline & "Fracture resistance" AND ("sealer" OR "endodontic filling" OR & 79 \\
& "Root Canal Obturation"[Mesh]) & \\
SciELO & "Fracture resistance" AND "sealer" AND "endodontically treated" & 43 \\
Trip Database & "Fracture resistance" AND ("sealer" OR "Root Canal Obturation") & 25 \\
LILACS & "Fracture resistance" AND ("sealer" OR "Root Canal Obturation") & 6 \\
Web of Science & "Fracture resistance" AND ("sealer" OR "Root Canal Obturation") & 33 \\
& AND "endodontically treated" & 16 \\
Cochrane & "Fracture resistance" AND ("sealer" OR "Root Canal Obturation") \\
\hline
\end{tabular}

\section{RESULTADOS}

Descripción de los estudios: La búsqueda inicial en las seis bases de datos (Medline, SciELO, Trip Database, LILACS, Web of science y Cochrane) dio como resultado un total de 202 artículos, de los cuales se excluyeron 67 por duplicados. El flujograma de la selección final de los artículos se detalla en la Figura 1. Ocho artículos se revisaron a texto completo y en esta etapa se excluyeron dos estudios: Uno que no utilizó sellador en base a resina epóxica como grupo comparación (Celikten et al., 2015) y otro por expresar los valores de resistencia a la fractura en MPa y no en Newtons, no pudiendo realizarse la comparación con los otros estudios (Mandava et al., 2014).

Un total seis estudios fueron incluidos en esta revisión (Sagsen et al., 2012; Topçuoglu et al., 2013; Ersoy \& Evcil, 2015; Sungur et al., 2016; Dibaji et al., 2017; Upadhyay et al.).

Las características y resultados de los estudios incluidos en esta revisión se detallan en la Tabla II.

Riesgo de sesgo en los estudios incluidos: A estos seis artículos se le aplicó la tabla de riesgo de sesgo previamente establecida, dando como resultado que dos estudios obtuvieron puntuación cuatro (Ersoy \& Evcil; Sungur et al.), y los restantes cuatro estudios obtuvieron puntaje cinco (Sagsen et al., 2012; Topçuoglu et al.; Dibaji et al.; Upadhyay et al.), por lo que todos los estudios incluidos obtuvieron una calidad moderada (Tabla III).

De los artículos incluidos en esta revisión, se analizaron de forma in vitro un total de 420 dientes entre incisivos y premolares unirradiculares. Los cementos selladores usados para la obturación de los canales radiculares en conjunto con conos de gutapercha fueron los siguientes: En el grupo de los selladores en base a resinas epóxica: $\mathrm{AH}$ plus, $\mathrm{AH}$ plus jet \& AH26, y en el grupo de los selladores en base a biocerámicos iRoot SP, MTA Fillapex, Endosequence $B C$, Tech Biosealer Endo, Endoseal MTA. En cuanto a los resultados de los seis artículos en ninguno se encontraron diferencias significativas entre selladores en base de resina epóxica y selladores en base a biocerámicos en relación a la resistencia a la fractura.

Sin embargo en dos estudios (Topçuoglu et al.; Sungur et al.) se encontraron diferencias significativas en dos selladores biocerámicos, Tech Biosealer Endo y MTA Fillapex, los cuales tuvieron un peor desempeño en comparación a selladores en base a resina epóxica como con otros selladores biocerámicos.

Por otro lado, en cuatro estudios los valores promedios más altos estuvieron dados por el $\mathrm{AH}$ plus superando a los selladores biocerámicos (Sagsen et al., 2012; Ersoy \& Evcil; Dibaji et al.; Upadhyay et al.), y por su contraparte en dos estudios (Topçuoglu et al.; Sungur et al.) los selladores biocerámicos Endosequence BC y iRoot SP dieron valores promedios más altos que los selladores a base de resina epóxica.

\section{DISCUSIÓN}

Al analizar los estudios seleccionados se establece que en cuanto a resistencia a la fractura no hay diferencias significativas entre los dientes obturados con cementos selladores a base de biocerámicos y aquellos obturados con cementos selladores a base de resina epóxica. Sin embargo, llama la atención la diversidad de factores que podrían condicionar este resultado. Éstos tienen relación principalmente con la estandarización de las muestras, el tipo de instrumentación bioquimiomecánica, el sistema de obturación 


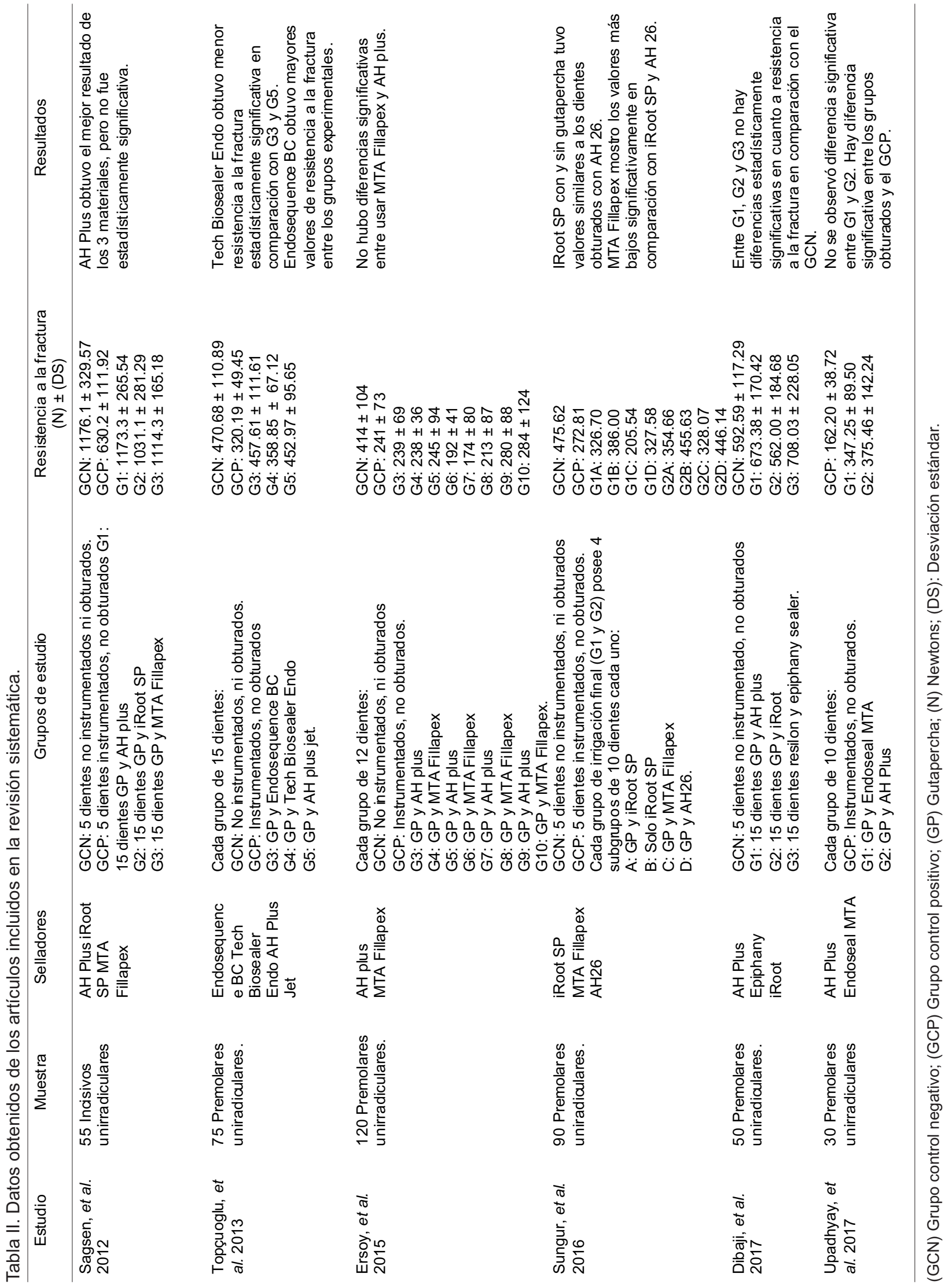


Tabla III. Análisis de riesgo de sesgo de los artículos incluidos.

\begin{tabular}{|c|c|c|c|c|c|c|c|c|}
\hline Estudios & $\begin{array}{l}\text { Aleatorización } \\
\text { de los dientes }\end{array}$ & $\begin{array}{c}\text { Dientes libres } \\
\text { de } \\
\text { caries/cracks }\end{array}$ & $\begin{array}{l}\text { Instru- } \\
\text { cciones del } \\
\text { fabricante }\end{array}$ & $\begin{array}{l}\text { Dientes con } \\
\text { dimensión } \\
\text { similar }\end{array}$ & $\begin{array}{l}\text { Cálculo de } \\
\text { tamaño } \\
\text { muestral }\end{array}$ & $\begin{array}{c}\text { Cegamient } \\
\text { o del } \\
\text { operador }\end{array}$ & $\begin{array}{c}\text { Medio de } \\
\text { conservación }\end{array}$ & $\begin{array}{l}\text { Riesgo de } \\
\text { sesgo }\end{array}$ \\
\hline $\begin{array}{l}\text { Sagsen, et } \\
\text { al. } 2012\end{array}$ & + & + & + & + & - & - & + & Moderado \\
\hline $\begin{array}{l}\text { Topçuoglu, } \\
\text { et al. } 2013\end{array}$ & + & + & + & + & - & - & + & Moderado \\
\hline $\begin{array}{l}\text { Ersoy, et al. } \\
2015\end{array}$ & + & + & - & + & - & - & + & Moderado \\
\hline $\begin{array}{l}\text { Sungur, et } \\
\text { al. } 2016\end{array}$ & + & + & - & + & - & - & + & Moderado \\
\hline $\begin{array}{l}\text { Dibaji, et al. } \\
2017\end{array}$ & + & + & + & + & - & - & + & Moderado \\
\hline $\begin{array}{l}\text { Upadhyay, et } \\
\text { al } 2017\end{array}$ & + & + & + & + & - & - & + & Moderado \\
\hline
\end{tabular}

seleccionado y el tipo de carga entre otros factores que se discuten a continuación.

Con respecto a la estandarización de las muestras, resulta difícil estandarizar los dientes humanos para evaluar la resistencia a la fractura debido a las variaciones anatómicas, edad de los pacientes o tiempo de extracción de los dientes. Cuando los dientes extraídos son testeados, se deben estandarizar factores como el ancho mesiodistal y bucolingual, así como la longitud de la raíz (Sagsen et al., 2007). En relación a lo anterior, de las 420 muestras incluidas en esta revisión, sólo 55 corresponden a incisivos unirradiculares pertenecientes al estudio de Sagsen et al. (2012). Los cinco estudios restantes, con un total de 365 muestras, utilizaron sólo premolares unirradiculares.

En situaciones clínicas, es el ligamento periodontal el cual se encarga de prevenir las concentraciones de estrés en regiones particulares y distribuye las cargas aplicadas a lo largo de toda la superficie radicular. De los estudios incluidos, tres simularon el ligamento periodontal y hueso alveolar mediante pasta de silicona y bloques de resina de poliestireno sosteniendo que la presencia de ligamento periodontal modifica el tipo de fractura, fracturando la raíz en diferentes lugares (Topçuoglu et al.; Ersoy \& Evcil; Upadhyay et al.). Los tres estudios restantes incluidos en esta revisión se limitan a un montaje de las muestras en cubos acrílicos lo cual podría haber influido en sus resultados.

En relación al tipo de instrumentación utilizada, en todos los estudios seleccionados fue realizada mediante el sistema de instrumentación rotatorio Protaper Universal (Dentsply Maillefer) el cual es conocido por producir preparaciones más redondas y uniformes al corte transversal, distribuyendo el estrés de manera más uniforme dentro de la raíz durante la obturación, reduciendo así la susceptibilidad a la fractura. Sin embargo, el efecto de los sistemas de instrumentación rotatorio en la dentina radicular es controversial, ya que se ha reportado un mayor riesgo asociado a líneas de fractura, cracks de dentina y mayor debilitamiento de las paredes del conducto en comparación al instrumental manual (Bier et al., 2009). En todos los estudios seleccionados se estandarizó la lima maestra a ProTaper F3 o F4, por lo que en cuanto al calibre de las preparaciones son muy similares en todos los dientes incluidos.

Otro punto a considerar son los cracks apicales, los cuales tienden a manifestarse en mayor medida cuando se realiza limpieza e instrumentación de toda la longitud radicular y en menor medida cuando se trabaja a $1 \mathrm{~mm}$ del ápex (Adorno et al., 2009). En relación a lo anterior, sólo el estudio de Ersoy \& Evcil trabajó las muestras a 0,5 mm del ápex, los cinco artículos restantes lo hicieron a $1 \mathrm{~mm}$ del ápex.

El tipo de irrigación también tiene influencia en la resistencia a la fractura. Tanto el uso de hipoclorito de sodio $(\mathrm{NaOCl})$ como de Ácido etilendiaminotetraacético (EDTA) en la irrigación del conducto radicular altera las propiedades físicas de la dentina disminuyendo la resistencia a la flexión, el módulo elástico, y la microdureza (Oliveira et al., 2007). Esta disminución en la resistencia a la fractura está directamente relacionada con el tiempo y la concentración del irrigante, sin embargo, no existe un protocolo de irrigación común a todos los artículos seleccionados. Sólo en el estudio de Sungur et al. se analizó la influencia de dos protocolos de irrigación distintos, uno con $\mathrm{NaOCl}$ y otro con clorhexidina $(\mathrm{CHX})$, en la resistencia a la fractura, donde concluyó que los 
grupos irrigados con $\mathrm{NaOCl}$ mostraron significativamente mayores niveles de fractura vertical que aquellos irrigados con $\mathrm{CHX}$.

De acuerdo al tipo de obturación utilizada, Shashidhar \& Shashidhar (2014), compararon la fuerza de fractura entre dientes obturados mediante técnica de condensación lateral y vertical. La resistencia a la fractura máxima se obtuvo obturando los conductos mediante la técnica de condensación lateral. Sólo tres estudios de esta revisión incluyen la técnica de condensación lateral en sus muestras (Sagsen et al. 2012; Ersoy \& Evcil; Dibaji et al.).

Con respecto al método para testear la resistencia a la fractura, todos los estudios utilizan la máquina de testeo universal, sin embargo, no existe una configuración estándar para sus variables. Estas son el ancho de la parte activa, velocidad y dirección en la que se aplica la fuerza. La mayoría de los estudios publicados utilizan cargas verticales aplicadas sobre el eje longitudinal del diente, ya que, de esta forma se transfiere la carga uniformemente a toda la raíz (Dias de Souza et al., 2002). Sin embargo, se ha reportado que la aplicación de una fuerza en diferentes ángulos en relación al eje mayor de un diente es más similar a la carga oclusal en condiciones clínicas (Loney et al., 1995). Excepcionalmente, en un estudio realizado por Sagsen et al. (2012) se aplicaron fuerzas en un ángulo de $45^{\circ}$ paralelo al eje axial del diente, esta variación en la angulación podría ser la causa de los altos valores de resistencia a la fractura registrados en comparación a estudios que aplican fuerzas paralelas al eje mayor del diente.

En relación al patrón de fractura, Zamin et al. (2012) afirmaron que la dirección de la aplicación de la fuerza puede influir en la dirección de las fracturas debido a que las fracturas hacia la región buccolingual se observan generalmente en estudios que aplican una fuerza vertical (Teixeira et al., 2004; Topçuoglu et al.). Topçuoglu et al. compararon tres selladores, dos biocerámicos y uno en base a resina epóxica, concluyendo que el tipo de sellador utilizado no tuvo impacto en el patrón de fractura.

En cuanto a comparaciones con grupos control de los estudios seleccionados, cinco estudios concluyen que el grupo control positivo, el cual es el diente instrumentado y no obturado, es el que presenta los valores más bajos en cuanto a resistencia a la fractura y el grupo control negativo, que corresponde a dientes no instrumentados y no obturados, los valores más altos. Dos de los estudios anteriores afirman además, que todos los selladores utilizados en sus muestras, sean biocerámicos o resinosos, son capaces de reforzar significativamente al grupo control positivo (instrumentado y no obturado) (Sagsen et al., 2012; Upadhyay et al.). Sin embargo, uno de los estudios seleccionados concluyó que no existen diferencias estadísticamente significativas al comparar el grupo control negativo (no instrumentados y no obturados) con los grupos obturados tanto con selladores biocerámicos como resinosos (Dibaji et al.).

La introducción de los selladores biocerámicos comenzó a ofrecer una nueva alternativa a los otros materiales de relleno en la obturación endodóntica. Dentro de los selladores a base de biocerámicos más estudiados en la actualidad se encuentran los que son a base de silicato cálcico y los que son a base de MTA. Aunque ambos selladores tienen propiedades similares tales como el contenido de silicato de calcio y la producción de hidroxiapatita durante la fase de fraguado, en el estudio de Topçuoglu et al. el uso del sellador biocerámico a base de MTA no aumentó significativamente la resistencia a la fractura, además fue el único del estudio en ser suministrado en forma de polvo y líquido. En el estudio de Upadhyay et al. se utilizó un sellador resinoso y otro biocerámico suministrados como pastas, los resultados mostraron que ambos selladores presentaron mayores valores de resistencia a la fractura. El hecho de que los selladores con una formulación de pasta aumenten la resistencia a la fractura puede ser debido al flujo más alto y a una mejor penetración en los túbulos dentinarios que los selladores con una formulación en polvo/líquido (Topçuoglu et al.). Estudios recientes han revelado que los nuevos selladores a base de materiales biocerámicos tienen fuerzas de unión y capacidad de sellado apical similares a los selladores en base a resina (Ersahan \& Aydin, 2010; Nagas et al., 2012).

Si bien la evidencia que pueden aportar los estudios in vitro es limitada, los resultados de estos son de utilidad como una aproximación más cercana a la realidad clínica, especialmente en cuanto a estudios de resistencia a la fractura, en donde no es factible utilizar otra metodología debido a razones éticas y prácticas. En relación a lo anterior, se requieren más estudios que comparen la resistencia a la fractura que otorgan los diferentes selladores biocerámicos. Además, es necesaria una estandarización en el tratamiento de las muestras, ya que, esto permitiría contrastar la evidencia actual con la de estudios previos con una menor cantidad de factores condicionantes. 


\section{CONCLUSIÓN}

Los artículos seleccionados, teniendo en consideración las limitaciones propias de los estudios in vitro, concluyen que en cuanto a resistencia a la fractura no hay diferencias significativas entre el uso de cementos selladores a base de biocerámicos y cementos selladores a base de resina epóxica en la obturación radicular de DTE.

Se requieren de más estudios in vitro con mayor estandarización y mayor calidad metodológica que comparen resistencia a la fractura de los cementos selladores, para así lograr obtener un criterio unánime al momento de la elección entre un cemento sellador a base de biocerámicos y uno a base de resina epóxica.

Al momento de elegir un cemento, el clínico debe basarse no sólo en una característica como la resistencia a la fractura, sino también en otras, como lo son la biocompatibilidad, tiempo de trabajo, solubilidad, costo, evalúando cada caso clínico en particular.

MORALES, C. L. F.; REYES, M. S. I.; ÁLVAREZ, V. S. J. \& HERNÁNDEZ-VIGUERAS, S. Fracture resistance of endodontically treated teeth obturated with bioceramic sealers and epoxy resin-based sealers: A systematic review. Int. J. Odontostomat., 13(1):31-39, 2019,

ABSTRACT: The objective of this study was to determine through the review of scientific literature, the type of sealer that provides the greatest resistance to fracture in endodontically treated teeth. Bioceramic sealer or epoxy resin based sealers were considered for this analysis. A systematic review was performed according to PRISMA, in the databases Medline, SciELO, Trip Database, LILACS, Web of Science, Cochrane. The search was carried out over the last 10 years, and only in vitro studies were included; 202 articles were found and subsequently, duplicate articles were eliminated, non-inferential studies by title and abstracts were excluded, leaving eight articles that were revised to full text. In this stage, two studies were excluded. In total, six studies were included in this review. Of these, none found significant difference between sealer cements based on bioceramics and based on epoxy resin, in terms of resistance to fracture of endodontically treated teeth. However, in 4 studies the highest found values of fracture resistance were given by sealers based on epoxy resin. Taking into account the limitations inherent to in vitro studies, this review concludes that in terms of fracture resistance, there are no significant differences between the use of sealer based on bioceramics and the based on epoxy resin in the root canal obturation.

KEY WORDS: bioceramics sealers, fracture resistance, endodontically treated teeth, root canal obturation.

\section{REFERENCIAS BIBLIOGRÁFICAS}

Adorno, C. G.; Yoshioka, T. \& Suda, H. The effect of root preparation technique and instrumentation length on the development of apical root cracks. J. Endod., 35(3):389-92, 2009.

Ashraf, H.; Momeni, G.; Moradi Majd, N. \& Homayouni, H. Fracture resistance of root canals obturated with Gutta-Percha versus Resilon with two different techniques. Iran. Endod. J., 8(3):1369, 2013.

Bier, C. A.; Shemesh, H.; Tanomaru-Filho, M.; Wesselink, P. R. \& Wu, M. K. The ability of different nickel-titanium rotary instruments to induce dentinal damage during canal preparation. J. Endod., 35(2):236-8, 2009.

Celikten, B.; Uzuntas, C. F. \& Gulsahi, K. Resistance to fracture of dental roots obturated with different materials. BioMed Res. Int., 2015:591031, 2015.

Chang, E.; Lam, E.; Shah, P. \& Azarpazhooh, A. Cone-beam computed tomography for detecting vertical root fractures in endodontically treated teeth: a systematic review. J. Endod., 42(2):177-85, 2016.

Dias de Souza, G. M.; Pereira, G. D.; Dias, C. T. \& Paulillo, L. A. Fracture resistance of premolars with bonded class II amalgams. Oper. Dent., 27(4):349-53, 2002.

Dibaji, F.; Afkhami, F.; Bidkhori, B. \& Kharazifard, M. J. Fracture Resistance of Roots after Application of Different Sealers. Iran. Endod. J., 12(1):50-4, 2017.

Ersahan, S. \& Aydin, C. Dislocation resistance of iRoot SP, a calcium silicate-based sealer, from radicular dentine. J. Endod., 36(12):2000-2, 2010

Ersoy, I. \& Evcil, M. S. Evaluation of the effect of different root canal obturation techniques using two root canal sealers on the fracture resistance of endodontically treated roots. Microsc. Res. Tech., 78(5):404-7, 2015.

García-Guerrero, C.; Parra-Junco, C.; Quijano-Guauque, S.; Molano, N.; Pineda, G. A. \& Marín-Zuluaga, D. J. Vertical root fractures in endodontically-treated teeth: A retrospective analysis of possible risk factors. J. Investig. Clin. Dent., 9(1), 2018.

Lam, P. P.; Palamara, J. E. \& Messer, H. H. Fracture strength of tooth roots following canal preparation by hand and rotary instrumentation. J. Endod., 31(7):529-32, 2005.

Loney, R. W.; Moulding, M. B. \& Ritsco, R. G. The effect of load angulation on fracture resistance of teeth restored with cast post and cores and crowns. Int. J. Prosthodont., 8(3):247-51, 1995.

Malhotra, S.; Hedge, M. \& Shetty, C. Bioceramic technology in endodontics. Br. J. Med. Med. Res., 4(12):2446-54, 2014.

Mandava, J.; Chang, P. C.; Roopesh, B.; Faruddin, M. G.; Anupreeta, A. \& Uma, Ch. Comparative evaluation of fracture resistance of root dentin to resin sealers and a MTA sealer: An in vitro study. J. Conserv. Dent., 17(1):53-6, 2014.

Moher, D.; Liberati, A.; Tetzlaff, J.; Altman, D. G. \& PRISMA Group. Preferred reporting items for systematic reviews and metaanalyses: the PRISMA statement. PLoS Med., 6(7):e1000097, 2009.

Nagas, E.; Uyanik, M. O.; Eymirli, A.; Cehreli, Z. C.; Vallittu, P. K.; Lassila, L. V. \& Durmaz, V. Dentin moisture conditions affect the adhesion of root canal sealers. J. Endod., 38(2):240-4, 2012.

Oliveira, L. D.; Carvalho, C. A.; Nunes, W.; Valera, M. C.; Camargo, C. H. \& Jorge, A. O. Effects of chlorhexidine and sodium hypochlorite on the microhardness of root canal dentin. Oral Surg. Oral Med. Oral Pathol. Oral Radiol. Endod., 104(4):e125-8, 2007.

Sagsen, B.; Er, O.; Kahraman, Y. \& Akdogan, G. Resistance to fracture of roots filled with three different techniques. Int. Endod. J., 40(1):31-5, 2007.

Sagsen, B.; Üstün, Y.; Pala, K. \& Demırbuga, S. Resistance to frac- 
MORALES, C. L. F.; REYES, M. S. I.; ÁLVAREZ, V. S. J. \& HERNÁNDEZ-VIGUERAS, S. Resistencia a la fractura de dientes tratados endodónticamente obturados con selladores biocerámicos versus selladores resinosos. Revisión sistemática. Int. J. Odontostomat., 13(1):31-39, 2019.

ture of roots filled with different sealers. Dent. Mater. J., 31(4):52832, 2012.

Sarkis-Onofre, R.; Skupien, J. A.; Cenci, M. S.; Moraes, R. R. \& Pereira-Cenci, T. The role of resin cement on bond strength of glass-fiber posts luted into root canals: a systematic review and meta-analysis of in vitro studies. Oper. Dent., 39(1):E31-44, 2014.

Schilder, H. Filling root canals in three dimensions. Dent. Clin. North Am., 723-44, 1967.

Sedrez-Porto, J. A.; Rosa, W. L.; da Silva, A. F.; Münchow, E. A. \& Pereira-Cenci, T. Endocrown restorations: A systematic review and meta-analysis. J. Dent., 52:8-14, 2016.

Shashidhar, J. \& Shashidhar, C. Gutta percha verses resilon: an in vitro comparison of fracture resistance in endodontically treated teeth. J. Indian Soc. Pedod. Prev. Dent., 32(1):53-7, 2014.

Silva Almeida, L.; Moraes, R. R.; Morgental, R. D. \& Pappen, F. G. Are premixed calcium silicate-based endodontic sealers comparable to conventional materials? A systematic review of in vitro studies. J. Endod., 43(4):527-35, 2017.

Sungur, D. D.; Altundasar, E.; Uzunoglu, E. \& Yilmaz, Z. Influence of different final irrigation regimens and various endodontic filling materials on vertical root fracture resistance. Niger. J. Clin. Pract., 19(2):267-71, 2016.

Teixeira, F. B.; Teixeira, E. C.; Thompson, J.; Leinfelder, K. F. \& Trope, M. Dentinal bonding reaches the root canal system. J. Esthet. Restor. Dent., 16(6):348-54, 2004.

Topçuoglu, H. S.; Tuncay, Ö.; Karatas, E.; Arslan, H. \& Yeter, K. In vitro fracture resistance of roots obturated with epoxy resin-based, mineral trioxide aggregate-based, and bioceramic root canal sealers. J. Endod., 39(12):1630-3, 2013.

Upadhyay, S. T.; Purayil, T. P. \& Ginjupalli, K. Comparative evaluation of Fracture resistance of endodontically treated teeth obturated with Pozzolan-based MTA sealer and epoxy resin-based sealer: An in vitro study. World J. Dent., 8(1):37-40, 2017.

Zamin, C.; Silva-Sousa, Y. T.; Souza-Gabriel, A. E.; Messias, D. F. \& Sousa-Neto, M. D. Fracture susceptibility of endodontically treated teeth. Dent. Traumatol., 28(4):282-6, 2012.
Dirección para correspondencia:

Scarlette Hernández-Vigueras.

Instituto de Odontoestomatología

Rudloff 1640

Valdivia

CHILE

Email: shernandez@uach.cl

Recibido : 13-07-2018

Aceptado: 18-10-2018 